\title{
DEXAMETASONA AL 0,1\% VERSUS RIMEXOLONA AL 1\%. ESTUDIO COMPARATIVO EN EL POSTOPERATORIO DE LA CIRUGÍA DE CATARATAS
}

\section{1\% DEXAMATHASONE AND 1\% RIMEXOLONE. A COMPARATIVE STUDY IN THE POSTOPERATIVE TREATMENT AFTER CATARACT EXTRACTION}

\author{
VICO-RUIZ E ${ }^{1}$, BENÍTEZ-DEL-CASTILLO-SÁNCHEZ JM ${ }^{2}$, CUIÑA-SARDIÑA R ${ }^{1}$, \\ MARTÍNEZ-DE-LA-CASA JM², GARCÍA-SÁNCHEZ J²
}

\section{RESUMEN}

Objetivo: Comparar la eficacia y los efectos secundarios de la rimexolona $1 \%$ y de la dexametasona $0,1 \%$ en el tratamiento postoperatorio de la cirugía de catarata.

Material y métodos: Estudio de cohortes prospectivo en el que se ha seleccionado una muestra de 37 pacientes intervenidos de cataratas mediante facoemulsificación, sin complicaciones intraoperatorias, en el Hospital Clínico San Carlos, y se han dividido en dos grupos. El grupo DEX, de 19 pacientes se trato con dexametasona al $0,1 \%$ tópica como tratamiento antiinflamatorio y el grupo RIMEX, de 18 pacientes se trató con rimexolona 1\% tópica siguiendo la misma pauta en ambos casos. En todos los pacientes se han estudiado la agudeza visual, la hiperemia conjuntival, las células en cámara anterior, el flare, la presión intraocular, el grosor corneal y la presencia de edema macular a las 24 horas y al mes de la cirugía.

\begin{abstract}
Purpose: To compare the efficiency and secondary effects of using $1 \%$ rimexolone or $0.1 \%$ dexamethasone as postoperative treatment for cataract surgery. Materials and methods: A prospective study performed on a cohort of 37 patients undergoing cataract surgery by phacoemulsification with no intraoperative complications at the Hospital Clínico San Carlos, Madrid. After surgery, 19 of the patients were randomly assigned to receive topical $0.1 \%$ dexamethasone (DEX group) as inflammatory treatment and the remaining 18 subjects were treated with $1 \%$ rimexolone ( RIMEX group) following the same regime. Twenty four hours and one month after surgery, visual acuity, conjunctival hyperaemia, anterior chamber cells, anterior chamber flare, intraocular pressure, corneal thickness and macular edema were determined in each patient.

Results: The repeated measures test performed on 24 hours and 1 month data revealed a significant
\end{abstract}

\footnotetext{
Recibido: 31/10/07. Aceptado: 26/6/09.

Hospital Clínico San Carlos. Madrid. España.

1 Licenciado en Medicina.

2 Doctor en Medicina.

Correspondencia:

Eva Vico Ruiz

Unidad de Superficie e Inflamación Ocular

Hospital Clínico Universitario de San Carlos

C/. Martín Lagos, s/n

28040 Madrid

España

E-mail: EBUSQUI@gmail.com
} 
Resultados: Al comparar ambos fármacos mediante el test de medidas repetidas a las 24 horas y al mes de la cirugía se obtuvieron diferencias estadísticamente significativas en el tyndall $(\mathrm{p}=0,001)$ y en el flare $(\mathrm{p}=0,034)$, siendo la reducción de éstos parámetros mayor en el grupo dexametasona, mientras que no se observaron cambios significativos en el resto de los parámetros evaluados.

Conclusiones: La Rimexolona ha resultado ser un fármaco útil y seguro, al igual que la dexametasona, como tratamiento postoperatorio de la cirugía de catarata.

Palabras clave: Rimexolona, dexametasona, cirugía de cataratas, hipertensión ocular. difference between the two treatments in terms of Tyndall $(\mathrm{p}=0.001)$ and flare $(\mathrm{p}=0.034)$ values; these variables being lower in the dexamethasone group. No differences were observed in the remaining variables examined.

Conclusions: Rimexolone is as efficient and safe as dexamethasone for the treatment of patients undergoing cataract extraction (Arch Soc Esp Oftalmol 2009; 84: 299-304).

Key words: Rimexolone, dexamethasone, cataract extraction, ocular hypertension.

\section{INTRODUCCIÓN}

Los corticoides son potentes agentes antiinflamatorios e inmunosupresores que actúan a diferentes niveles pero sobre todo en la inmunidad celular inhibiendo las enzimas y citokinas proinflamatorias responsables del proceso inflamatorio.

El uso tópico de corticoides produce menos efectos secundarios sistémicos que si se administran por vía sistémica. No obstante, el uso tópico puede producir efectos adversos oculares: catarata, aumento de presión intraocular (PIO) e infecciones. Los incrementos de la PIO son más frecuentes en pacientes con glaucoma primario de angulo abierto, diabetes y miopía, debido a que existe una dificultad para el flujo de drenaje del humor acuoso en estos individuos. Se sabe que en la malla trabecular de los pacientes respondedores a corticoides existe un material extracelular semejante al de la membrana basal que podría ser el responsable del incremento de la PIO (1).

Existen diferentes tipos de corticoides con distinta potencia. Entre los menos potenes se encuentra la fluorometolona. Otros con mayor potencia como la dexametasona o el acetato de prednisolona tienen mayor efecto antiinflamatorio, sin embargo producen mayores aumentos de la presión intraocular $(2,3)$.

El corticoide ideal sería aquel que con una buena potencia antiinflamatoria produjese los mínimos efectos adversos posibles. En este sentido, la rimexolona ha demostrado poseer una buena potencia antiinflamatoria, comparable a la del acetato de prednisolona $1 \%$ (4) y a la de la dexametasona, sin producir incrementos de la PIO. Por otro lado, la rimexolona carece de capacidad para suprimir el eje pituitario-adrenal que tiene lugar con el uso de otros corticoides (5).

La estructura de la rimexolona oftálmica es algo diferente a la de otros antiinflamatorios corticoideos. Posee su núcleo esteroideo con modificaciones tales como un anillo en las posiciones 1-2 y 4-5 así como la presencia de grupos 3-ceto, 11b-hidroxilo, $16 a-m e t i l$ y 20 -ceto (11b-hidroxi-16a fluoro-6ametilprednisolona). Se añade una única modificación más, que consiste en que en las posiciones 17 a y 21 se substituye el grupo hidroxilo por metilo.

Diversos factores pueden contribuir a que la rimexolona tenga una actividad antiinflamatoria potente sin producir incrementos importantes de la PIO. Entre ellos está el tener una actividad glucocorticoide intrínseca, su penetración ocular limitada y su vida media biológica dentro del ojo (3). Su capacidad antiinflamatoria se debe fundamentalmente a una baja solubilidad acuosa y alta lipofílicidad. Además la novedosa estructura molecular le permite tener una acción tisular selectiva dentro del ojo con baja afinidad sobre el tejido trabecular por lo que la dificultad al drenaje del humor acuoso se va a ver muy reducida.

La rimexolona ha demostrado ser de utilidad no solo como tratamiento postoperatorio de la cirugía de catarata sino también como tratamiento para otras patologías como la uveitis anterior (3) y la conjuntivitis alérgica (6).

El objetivo de este estudio es comparar la eficacia y seguridad de la rimexolona $1 \%$ con la de la dexametasona $0,1 \%$, el corticoide más ampliamente utilizado como tratamiento antiinflamatorio tras la cirugía de la catarata. 


\section{SUJETOS, MATERIAL Y MÉTODOS}

Se ha realizado un estudio de cohortes prospectivo. Se han seleccionado 37 pacientes consecutivos intervenidos de catarata no complicada mediante facoemulsificación con implante de lente intraocular en saco. No se han incluído pacientes con antecedentes personales o familiares de glaucoma, historia de diabetes mellitus ni miopes magnos con el fin de obviar aquellos individuos con propensión a tener hipertensión ocular secundaria al uso de corticoides. Igualmente a aquellos con antecedentes personales de uveitis, inflamaciones de la superficie ocular, o con enfermedades sistémicas inflamatorias importantes (como artritis reumatoide, lupus, etc.) para evitar en ellos el riesgo de reacciones inflamatorias postoperatorias más severas de lo habitual. No se han incluido en el estudio pacientes con cataratas hipermaduras, rotura de cápsula posterior intraoperatoria o hifemas postquirúrgicos.

Todos los pacientes firmaron un consentimiento informado y en todos los casos la intervención se realizó con anestesia tópica (Clorhidrato de tetracaina $1 \mathrm{mg} / \mathrm{ml}$ y clorhidrato de oxibuprocaína $4 \mathrm{mg} / \mathrm{ml}$ ). Durante la cirugía el ojo se irrigó con solución salina balanceada y se utilizó como viscoelástico el hialuronato sódico. Como tratamiento preoperatorio profiláctico se administró Povidona Iodada diluida al 5\% en fondos de saco.

Los pacientes se han dividido en dos grupos de forma aleatorizada:

- Grupo-DEX: 19 individuos a los que se les administró como tratamiento postoperatorio Dexametasona $0,1 \%$ y Tobramicina $\left(\right.$ Tobradex $^{\circledR}$, AlconCusi, Barcelona ) una gota cada 3 horas la primera semana tras la cirugía, empezando el mismo día de la intervención, y posteriormente se fue bajando la dosis cada cinco días hasta su suspensión pasando por pautas de una gota cada 4 horas, cada 6 horas, cada 8 horas, cada 12 horas y cada 24 horas.

- Grupo-RIMEX: 18 individuos a los que se les administró postoperatoriamente una gota de Tobramicina y otra de rimexolona $1 \%\left(\operatorname{Vexol}^{\circledR}\right.$, Alcon Cusi, Barcelona) cada 3 horas la primera semana tras la cirugía, también empezando el mismo día de la intervención, y la pauta de descenso del tratamiento fue igual que en el primer grupo hasta la suspensión. A todos los pacientes de éste segundo grupo se les advirtió que debían esperar cinco minutos entre la instilación de la gota de rimexolona y la de tobramicina.
Todos los pacientes fueron examinados por un mismo explorador (EV) y se les evaluó el ojo intervenido en dos ocasiones, a las 24-48 horas de la cirugía y al mes de la misma, estudiando los siguientes parámetros:

- La agudeza visual en escala de Snellen y la logarítmica de Bayley-Lovie.

- Exploración biomicroscópica: se cuantificó el grado de hiperemia de la conjuntiva bulbar en una escala de 0 a 3 cruces $(0=$ no hiperemia, $1=$ leve, $2=$ moderada y $3=$ intensa) y las células en cámara anterior que también se cuantificó entre 0 y 4 cruces $(0=$ no células an cámara anterior, $1=$ menos de 10 células/campo de $1 \mathrm{x} 1 \mathrm{~mm}, 2=$ entre 10-20 células/campo de $1 \times 1 \mathrm{~mm}, 3=$ de 20 a 50 células/campo de $1 \times 1 \mathrm{~mm}$, $4=$ más de 50 células/ campo de $1 \times 1 \mathrm{~mm}$ ).

- Flare. Se utilizó el flare meter (FM-500, Kowa, Japan). Se realizaron cinco medidas del flare para cada determinación y se obtuvo la media para el análisis estadístico medido en fotones por milisegundo.

- Presión intraocular (PIO) medida con el tonómetro de Perkins en milímetros de mercurio.

- Grosor corneal central con paquimetría ultrasónica (Paradigm, Paradigm Medical Industrie, Inc Model P55, USA) medida en micras para poder realizar la corrección de la PIO en función del espesor corneal.

- Tomografía de coherencia óptica macular (Stratus OCT, Zeiss, Alemania) donde valoramos el espesor macular medido en micras y la presencia o ausencia de edema macular.

Las variables se expresan mediante su media y desviación estándar (DE). En todos los casos se comprobó la distribución de la variable frente a los modelos teóricos y se contrastó la hipótesis de homogeneidad de variancias. Para el estudio comparativo (24 horas vs 1 mes) de los dos fármacos se aplico el test t de Student para datos pareados. Se realizó un test de medidas repetidas (24 horas y 1 mes) para determinar las diferencias de medias debido al efecto individual en el seguimiento ( 24 horas vs 1 mes), y entre fármacos. Para todos los contrastes se rechazó la hipótesis nula con un nivel alfa de 0,05.

El análisis se realizó con con el paquete estadístico SPSS ver 11.5 (SPSS Inc., Chicago, Illinois, USA).

\section{RESULTADOS}

De los 37 pacientes incluidos en el estudio 35\% eran hombres y $65 \%$ eran mujeres. La edad media 
en el grupo Grupo-DEX era de 76,8 DE 9,0 y en el grupo tratado con rimexolona Grupo-RIMEX de 70,5 DE 7,5 ( $\mathrm{p}=0,06)$.

No se encontraron diferencias estadísticamente significativas entre el Grupo-DEX y el GrupoRIMEX en la visita de las 24 horas en ninguno de los parámetros estudiados (tabla I).

Al comparar estos parámetros en el Grupo-DEX a las 24 horas y al mes encontramos mejoría estadísticamente significativa en la hiperemia, las células en cámara anterior, el flare y la PIO mientras que no existió diferencia estadísticamente significativa en la agudeza visual, paquimetría ni espesor macular. En el Grupo- RIMEX encontramos mejoría significativa entre la exploración de las 24 horas y la del mes en: la hiperemia, las células en cámara anterior, PIO y grosor corneal, mientras que no existió diferencia estadísticamente significativa en la agudeza visual, el flare ni en el espesor macular (tabla I).
Al comparar ambos fármacos mediante el test de medidas repetidas a las 24 horas y al mes de la cirugía se obtuvieron diferencias estadísticamente significativas en el tyndall $(\mathrm{p}=0,001)$ y en el flare $(\mathrm{p}=0,034)$, siendo la reducción de estos parámetros mayor en el Grupo-DEX, mientras que no se observaron cambios significativos en el resto de los parámetros evaluados (tabla II).

\section{DISCUSIÓN}

Tras una cirugía de catarata sobreviene de forma inevitable una inflamación intraocular. Aunque en muchos casos esta inflamación es autolimitada, en ocasiones, si no se ponen los medios para disminuirla ésta puede conducir a una serie de daños que pueden causar una disminución de la agudeza visual en algunos casos irreversible. La inflamación

Tabla I. Medias y desviaciones estándar a las 24 horas de la intervención y al mes en ambos grupos

\begin{tabular}{|c|c|c|c|c|c|c|}
\hline \multirow[t]{2}{*}{ Variables de eficacia } & \multicolumn{3}{|c|}{ Grupo-DEX } & \multicolumn{3}{|c|}{ Grupo- RIMEX } \\
\hline & 24 horas & $1 \mathrm{mes}$ & $\mathrm{P}$ & 24 horas & $1 \mathrm{mes}$ & $\mathrm{p}$ \\
\hline Agudeza Visual & $0,34 \mathrm{DE} 0,18$ & $0,31 \mathrm{DE} 0,16$ & 0,589 & 0,34 DE 0,19 & 0,22 DE 0,18 & 0,062 \\
\hline Hiperemia & 1,37 DE 0,49 & 0,00 DE 0,00 & 0,000 & 1,33 DE 0,48 & 0,05 DE 0,23 & 0,000 \\
\hline Tyndall & 2,37 DE 0,89 & 0,15 DE 0,37 & 0,000 & 1,80 DE 0,82 & 0,75 DE 0,73 & 0,000 \\
\hline Flare & 14,4 DE 8,9 & 7,7 DE 3,7 & 0,002 & $10,1 \mathrm{DE} 5,1$ & 8,5 DE 2,9 & 0,283 \\
\hline PIO & $19,6 \mathrm{DE} 4,1$ & $14,1 \mathrm{DE} 3,9$ & 0,000 & 22,4 DE 5,5 & 13,7 DE 2,7 & 0,000 \\
\hline Espesor macular & $220 \mathrm{DE} 43$ & 202 DE 24 & 0,058 & $195 \mathrm{DE} 46$ & $187 \mathrm{DE} 31$ & 0,401 \\
\hline Espesor corneal & $567 \mathrm{DE} 49$ & 537 DE 39 & 0,073 & 589 DE 53 & 553 DE 39 & 0,012 \\
\hline
\end{tabular}

PIO: presión ocular. Significación estadística de los cambios observados (p). Unidades de medición según se expone en material y métodos. DE: desviación estándar.

Tabla II. Medias, desviaciones estándar e intervalos de confianza del $95 \%$ a las 24 horas y al mes de la cirugía en el grupo de pacientes tratados con dexametasona y rimexolona

\begin{tabular}{|c|c|c|c|c|c|}
\hline & \multicolumn{2}{|c|}{24 horas } & \multicolumn{2}{|c|}{$1 \mathrm{mes}$} & \multirow[t]{2}{*}{$\mathrm{p}$} \\
\hline & Grupo-DEX & Grupo-RIMEX & Grupo-DEX & Grupo-RIMEX & \\
\hline Agudeza Visual & $\begin{array}{c}0,34 \text { DE } 0,18 \\
(0,25-0,42)\end{array}$ & $\begin{array}{c}0,34 \text { DE } 0,19 \\
(0,25-0,42)\end{array}$ & $\begin{array}{c}0,73 \text { DE } 0,24 \\
(0,61-0,84)\end{array}$ & $\begin{array}{c}0,59 \text { DE } 0,23 \\
(0,48-0,71)\end{array}$ & 0,452 \\
\hline Hiperemia & $\begin{array}{c}1,37 \text { DE } 0,49 \\
(1,14-1,59)\end{array}$ & $\begin{array}{c}1,33 \text { DE } 0,48 \\
(1,09-1,56)\end{array}$ & $\begin{array}{c}0,00 \text { DE } 0,00 \\
(-0,07-0,07)\end{array}$ & $\begin{array}{l}0,05 \text { DE } 0,23 \\
(-0,02-0,13)\end{array}$ & 0,265 \\
\hline Células en cámara anterior & $\begin{array}{c}2,37 \text { DE } 0,89 \\
(1,96-2,77)\end{array}$ & $\begin{array}{c}1,80 \text { DE } 0,82 \\
(1,39-2,22)\end{array}$ & $\begin{array}{c}0,15 \text { DE } 0,37 \\
(-0,11-0,43)\end{array}$ & $\begin{array}{c}0,75 \text { DE } 0,73 \\
(0,47-1,02)\end{array}$ & 0,001 \\
\hline Flare & $\begin{array}{c}14,4 \text { DE } 8,9 \\
(11,1-17,8)\end{array}$ & $\begin{array}{c}10,1 \text { DE } 5,1 \\
(6,5-13,5)\end{array}$ & $\begin{array}{c}7,7 \text { DE } 3,7 \\
(6,1-9,3)\end{array}$ & $\begin{array}{c}8,5 \text { DE } 2,9 \\
(6,9-10,1)\end{array}$ & 0,034 \\
\hline $\mathrm{PIO}$ & $\begin{array}{c}19,6 \text { DE } 4,1 \\
(17,7-21,9)\end{array}$ & $\begin{array}{c}22,4 \text { DE } 5,5 \\
(20,1-24,7)\end{array}$ & $\begin{array}{c}14,1 \text { DE } 3,9 \\
(12,4-15,6)\end{array}$ & $\begin{array}{l}13,7 \text { DE } 2,7 \\
(12,1-15,4)\end{array}$ & 0,123 \\
\hline Espesor Macular & $\begin{array}{c}220 \mathrm{DE} 43 \\
(199-241)\end{array}$ & $\begin{array}{r}195 \mathrm{DE} 46 \\
(173-216)\end{array}$ & $\begin{array}{c}202 \mathrm{DE} 24 \\
(189-215)\end{array}$ & $\begin{array}{l}187 \text { DE } 31 \\
(174-200)\end{array}$ & 0,421 \\
\hline Espesor Corneal & $\begin{array}{c}567 \mathrm{DE} 49 \\
(540-594)\end{array}$ & $\begin{array}{c}589 \text { DE } 53 \\
(562-615)\end{array}$ & $\begin{array}{c}537 \text { DE } 39 \\
(516-558)\end{array}$ & $\begin{array}{c}553 \text { DE } 39 \\
(532-573)\end{array}$ & 0,790 \\
\hline
\end{tabular}

Significación estadística de los cambios observados mediante el test de medidas múltiples (p). DE: desviación estándar. 
puede producir: daño del nervio óptico secundario al aumento de la presión intraocular, la pérdida de transparencia corneal relacionada con un edema corneal y una disminución de agudeza visual asociada a un edema macular crónico (7).

El tratamiento postoperatorio más extendido en la actualidad con el fin de disminuir dicha inflamación y sus posibles secuelas es el uso de corticoides tópicos. Los corticoides previenen y disminuyen los síntomas y signos de la inflamación postoperatoria, entre ellos la hiperemia ocular, la fotofobia, el tyndall, la dilatación de capilares y la presencia de proteínas en cámara anterior (8).

La amplia utilidad clínica de los corticoides está en algunos casos limitada por sus efectos secundarios como el incremento de la presión intraocular, particularmente con la dexametasona (2). Ya en 1963, Armaly estudió la respuesta hipertensiva ocular en adultos sanos a la administración de corticoides tópicos. Demostró que se producía un aumento de la PIO y una disminución del flujo de drenaje de humor acuoso en estos individuos tras 4 semanas de tratamiento. Así clasificó a los individuos estudiados en tres grupos de «respondedores» de acuerdo con el grado de respuesta que mostraban al tratamiento: Los respondedores bajos que experimentaban un aumento de la PIO de menos de $6 \mathrm{mmHg}$, los respondedores medios que veían aumentada su PIO entre 6 y $15 \mathrm{mmHg}$ y los respondedores altos en los que aumentaba por encima de $15 \mathrm{mmHg}$. Estimó que en la población general sana el 5\% son respondedores altos, el 35\% intermedios y el $60 \%$ respondedores bajos (9). En un intento por conseguir nuevos tipos corticoides con una capacidad antiinflamatoria efectiva sin que ello asocie una tendencia a la hipertensión ocular, han aparecido fármacos como la rimexolona. Se ha visto que la rimexolona posee una actividad antiinflamatoria mayor que la de la fluorometolona $0,1 \%$ y similar a la del acetato de prednisolona $1 \%$ (10) o a la de la dexametasona (11). Sin embargo tiene una propensión comparable a la de la fluorometolona a aumentar la PIO $(12,13)$ y por lo tanto mucho menor que la de la dexametasona y el acetato de prednisolona $1 \%$ $(3,5,11)$.

La eficacia de la rimexolona ha sido demostrada como tratamiento postoperatorio de la cirugía de cataratas. Lehmann $\mathrm{R}$ et al, compararon la rimexolona con un placebo, que contenía su vehículo, como tratamiento postcirugía de catarata y observaron que la rimexolona era más efectiva que el placebo en el control de la inflamación postoperatoria valorado por el tyndall y el flare en cámara anterior (14).

Assill K et al (15) y Bron (16) obtuvieron resultados similares a los anteriores en cuanto a la superioridad de la rimexolona frente al placebo en el control de la inflamación de la cámara anterior. Otros estudios han comparado la eficacia y seguridad de la rimexolona al $1 \%$ con la de la dexametasona al $1 \%$ en el postoperatorio de la cirugía de catarata sin encontrar diferencias estadísticamente significativas en su poder antiinflamatorio (17). En nuestro estudio la respuesta antiinflamatoria (tyndall y flare en cámara anterior) de la rimexolona ha resultado algo inferior a la dexametasona. Sin embargo, esta diferencia no ha tenido repercusión clínica, no ha incrementado la presión intraocular (glaucoma inflamatorio) ni el edema corneal ni macular en los dos grupos estudiados. Aún más, la agudeza visual final en el grupo-RIMEX es superior, aunque no estadísticamente significativa, que al del grupo-DEX.

Como conclusión diremos que la rimexolona es un fármaco seguro y eficaz en el tratamiento postoperatorio de la cirugía de cataratas. Aunque su potencia es algo inferior a la dexametasona, ésta es adecuada para el control de la inflamación postoperatoria que tiene lugar tras una intervención no complicada y en pacientes sin factores de riesgo que predispongan a inflamaciones postoperatorias superiores a lo habitual. La rimexolona aporta la ventaja de tener menos efectos secundarios potenciales que la dexametasona con casi los mismos beneficios en casos no complicados, por lo que puede resultar especialmente útil en aquellos pacientes respondedores a corticoides que han de someterse a este tipo de intervención dada la menor propensión de la rimexolona a producir incrementos de PIO asociados al tratamiento corticoideo en estos individuos. No obstante serían necesarios nuevos estudios clínicos con este tipo de pacientes para conocer con certeza el papel de la rimexolona como tratamiento postoperatorio de la catarata bajo estas circunstancias.

\section{BIBLIOGRAFÍA}

1. Rohen JW, Linner E, Witner R. Electron microscopic studies on the trabecular meshwork in two cases of corticosteroid glaucoma. Exp Eye Res 1973; 17: 1931.

2. Akingbehin AO. Comparative study of the intraocular pressure effects of fluorometholone 0,1\% versus dexamethasone 0.1\%. Br J Ophthalmol 1983; 67: 661-663. 
3. Foster CS, Alter $G$, DeBarge LR, Raizman MB, Crabb JL, Santos CI, et al. Efficacy and safety of rimexolone $1 \%$ ophthalmic suspension vs $1 \%$ prednisolone acetate in the treatment of uveitis. Am J Ophthalmol 1996; 122: 177-182.

4. Leibowitz, HM, Kupferman A. Bioavailability and therapeutic effectiveness of topically administered corticosteroids. Trans Am Acad Ophthalmol Otolaryngol 1975; 79: 78-88.

5. Leibowitz, HM, Bartlett JD, Rich R, McQuirter H, Stewart $R$, Assil K. Intraocular pressure-raising potential of $1.0 \%$ rimexolone in patients responding to corticosteroids. Arch Ophthalmol 1996; 114: 993-997.

6. Abelson M, George M, Drake M. Evaluation of rimexolone ophthalmic suspension in the antigen challenge model of allergic conjunctivitis. Invest Ophthalmol Vis Sci 1992; 33: 1112 .

7. The Miami Study Group. Cystoid macular edema in aphakic and pseudophakic eyes. Am J Ophthalmol 1979; 88: 45-48.

8. Ellis PP. Pharmacological effects of corticosteroids. Int Ophthalmol Clin 1966; 6: 799-819.

9. Armaly MF. Statistical attributes of the steroid hypertensive response in the clinically normal eye, I: tje demostration of three level of response. Invest Ophthalmol Vis Sci 1963; 4: 187-197.

10. Leibowitz HM, Kupferman A. Bioavailability and therapeutic effectiveness of topically administered corticosteroids. Trans Am Acad Ophthalmol Otolaryngol 1975; 79: 78-88.
11. Brogden RN, Wagstaff AJ. Rimexolone. A Review of its pharmacological properties and clinical potential in the management of ocular inflamation. BioDrugs 1997; 8: 68-79.

12. Solomon KD, Vroman DT, Barker D, Gehlken J. Comparison of ketorolac tromethamine $0.5 \%$ and rimexolone $1 \%$ to control inflamation after cataract extraction: prospective randomized double-masked study. J Cataract Refract Surg 2001; 27: 1232-1237.

13. Mindel J, Tavitian H, Smith H, Walker E. Comparative ocular pressure elevation by medrysone, fluorometholone, and dexamethasone phosphate. Arch Ophthalmol 1980; 98: 1577.

14. Lehmann R, Assil K, Stewart R, Fox K. Comparison of rimexolone $1 \%$ ophthalmic suspension to placebo in control of postcataract surgery inflammation. Invest Ophthalmol Vis Sci 1995; 36: 793.

15. Assil KK, Massry G, Lehmann R, Fox K, Stewart R. Control of ocular inflammation after cataract extraction with rimexolone 1\% opthalmic suspension. J Cataract Refract Surg 1997; 23: 750-757.

16. Bron A, Denis T, Hoang-Xuan T, Boureau-Andrieux C, Crozafon $P$, Hachet $E$, et al. The effects of rimexolone $1 \%$ in postoperative inflamation after cataract extraction. A double-masked placebo-controlled study. Eur J Ophthalmol 1998; 8: 16-21.

17. Ertugrul G, Karakücük S, Öztürk A, Baykal E, Haytoglu T. Comparison of rimexolone $1 \%$ ophthalmic suspension with dexamethasone $0.1 \%$ in control of postcataract surgery inflammation. Erciyes Medical Journal 2002; 24: 112. 